\title{
Biosynthesis of heparin
}

\section{Relationship between the polymerization and sulphation processes}

\author{
Kerstin LIDHOLT, ${ }^{*}$ Lena KJELLÉN and UIf LINDAHL \\ Department of Veterinary Medical Chemistry, The Swedish University of Agricultural Sciences, The Biomedical Center, \\ Box 575, S-751 23 Uppsala, Sweden
}

\begin{abstract}
Incubation of a mouse mastocytoma microsomal fraction with UDP- $\left[{ }^{3} \mathrm{H}\right] \mathrm{GlcA}$ and UDP-GlcNAc yielded proteoglycans containing non-sulphated polysaccharide chains. Similar incubations performed in the presence of the sulphate donor 3'-phosphoadenosine 5'-phosphosulphate (PAPS) produced both sulphated and non-sulphated proteoglycans, which were separated by chromatography on DEAE-cellulose Analysis by gel chromatography of single polysaccharide chains, released from the proteoglycans by alkali treatment, showed that the non-sulphated chains produced during incubation for $5 \mathrm{~min}$ or $25 \mathrm{~min}$, either in the absence or in the presence of PAPS, were of fairly small molecular size, with an average peak $M_{\mathrm{r}}$ of approx. $10 \times 10^{3}-15 \times 10^{3}$. In contrast, the sulphated chains exceeded $M_{\mathrm{r}} 100 \times 10^{3}$. Pulse-chase experiments suggested that sulphated chains were capable of further elongation. These results indicate that sulphation promotes, by so far unknown mechanisms, further chain elongation. Sulphated proteoglycan (retarded on DEAE-cellulose chromatography) isolated after similar incubation of the microsomal fraction for $1 \mathrm{~min}$ only was found to contain a mixture of sulphated and virtually non-sulphated polysaccharide chains. However, when $\left[{ }^{35} \mathrm{~S}\right] \mathrm{PAPS}$ was included in the incubations, some ${ }^{35} \mathrm{~S}$ was found to be associated, essentially as $N$-sulphate groups, also with the latter type of chains, preferentially the high- $M_{\mathrm{r}}$ fraction. These results are interpreted in terms of a biosynthetic model by which the heparin proteoglycan is generated through transient interactions of macromolecular intermediates with distinctly separate complexes of membranebound enzymes.
\end{abstract}

\section{INTRODUCTION}

The polysaccharide heparin is synthesized as a proteoglycan in connective-tissue-type mast cells. The process is initiated by the formation of a polypeptide core (Rodén, 1980), characterized by the presence of an extended internal sequence of alternating serine and glycine units (Robinson et al., 1978; Kjellén et al., 1989). After substitution of these serine residues with neutral galactosyl-galactosyl-xylosyl trisaccharide sequences, polysaccharide chain formation occurs by alternating transfer of D-glucuronic acid and $N$-acetyl-D-glucosamine units from the corresponding UDP-sugar precursors to the non-reducing termini of the nascent polymer. The resulting polysaccharide chains are converted into the final biosynthetic product, i.e. the heparin proteoglycan, through a series of modification reactions that include deacetylation of $N$-acetylglucosamine residues, sulphation of the resulting free amino groups, C-5-epimerization of D-glucuronic acid to L-iduronic acid units and finally $O$-sulphation at various positions (Kusche et al., 1988; for reviews see also Lindahl et al., 1986; Lindahl \& Kjellén, 1987; Lindahl, 1989). The reactions occur rapidly, the entire series of modifications being completed within $1 \mathrm{~min}$ for an individual polysaccharide chain (Höök et al., 1975), yet proceed in a stepwise manner, such that certain structural modifications are introduced before the subsequent reactions can be initiated (Jacobs- son \& Lindahl, 1980; Riesenfeld et al., 1980, 1982; Jacobsson et al., 1979, 1984).

The biosynthetic process outlined above has been elucidated largely through studies with cell-free systems, with the use of a mouse mastocytoma microsomal fraction as a source of proteoglycan precursor and biosynthetic enzymes. When such a preparation is incubated with the appropriate sugar nucleotides, UDPGlcA and UDP-GlcNAc, a non-sulphated proteoglycan is generated, the constituent polysaccharide chains of which are composed of alternating glucuronic acid and glucosamine units (Silbert, 1963; Lindahl et al., 1973; Lidholt et al., 1988). A fraction of the latter moieties have undergone $N$-deacetylation and thus carry unsubstituted amino groups. All subsequent polymermodification reactions require the presence of the sulphate donor 3'-phosphoadenosine 5'-phosphosulphate (PAPS). The studies cited generally involved experiments in which polymerization and polymer modification were conducted separately, by adding PAPS to microsomal preparations containing preformed (radiolabelled) non-sulphated polysaccharide chains (bound to proteoglycan). The present paper describes experiments in which polymerization and sulphation were performed simultaneously. The results showed unexpectedly that the two processes are mutually interdependent; not only is polymerization prerequisite to sulphation, but the latter reaction also promotes chain 
elongation, resulting in the formation of polysaccharide chains that are much longer than those obtained in the absence of sulphation.

\section{EXPERIMENTAL}

\section{Materials}

A microsomal fraction was prepared as described previously (Jacobsson et al., 1979) from a transplantable mouse mastocytoma (Furth et al., 1957). UDP$\left[{ }^{3} \mathrm{H}\right] \mathrm{GlcA}$ was synthesized enzymically from D-[1$\left.{ }^{3} \mathrm{H}\right]$ glucose $(15 \mathrm{Ci} / \mathrm{mmol}$; New England Nuclear) as reported previously (Jacobsson et al., 1979). Unlabelled UDP-GlcA and UDP-GlcNAc were obtained from Sigma Chemical Co. Unlabelled and ${ }^{35}$ S-labelled PAPS were prepared as described by Jacobsson et al. (1979) and by Jacobsson \& Lindahl (1980) respectively. The glycosaminoglycan preparations used (hyaluronan, chondroitin sulphate and heparin) were as described previously (Enerbäck et al., 1985).

\section{Methods}

Labelled polysaccharide chains were generated by incubating $50 \mu \mathrm{Ci}$ (approx. $3 \mathrm{nmol}$ ) of UDP- $\left[{ }^{3} \mathrm{H}\right] \mathrm{GlcA}$ and various amounts of UDP-GlcNAc and PAPS (labelled or unlabelled) with approx. $10 \mathrm{mg}$ of microsomal protein $/ \mathrm{ml}$ of $10 \mathrm{mM}-\mathrm{MnCl}_{2} / 10 \mathrm{mM}-\mathrm{MgCl}_{2} /$ $5 \mathrm{~mm}-\mathrm{CaCl}_{2} / 50 \mathrm{~mm}$-Hepes buffer, $\mathrm{pH}$ 7.4. After incubation at $37^{\circ} \mathrm{C}$ for the periods of time indicated the reactions were interrupted by the addition of equal volumes of $4 \%(\mathrm{w} / \mathrm{v}) \mathrm{SDS}$, and the mixtures were then heated at $100{ }^{\circ} \mathrm{C}$ for $3 \mathrm{~min}$. After the addition of $0.5 \mathrm{mg}$ of carrier heparin, the incubation mixtures $(0.2-1 \mathrm{ml})$ were passed through columns $(1 \mathrm{~cm} \times 35 \mathrm{~cm})$ of Sephadex G-50 (medium grade; Pharmacia), equilibrated with $0.1 \mathrm{~m}-\mathrm{NaCl} / 0.1 \% \mathrm{SDS} / 50 \mathrm{~mm}$-Tris/HCl buffer, $\mathrm{pH} 8.0$. Fractions $(2 \mathrm{ml})$ were collected at a rate of $12 \mathrm{ml} / \mathrm{h}$ and analysed for radioactivity by liquid-scintillation counting; labelled proteoglycan was recovered in a distinct peak at the excluded volume of the columns.

Conversion of proteoglycans into single polysaccharide chains was achieved by adding $\mathrm{NaOH}$ to a final concentration of $0.5 \mathrm{M}$. The alkaline samples were kept at room temperature for $20 \mathrm{~h}$ and were then neutralized by the addition of $\mathrm{HCl}$.

Ion-exchange chromatography of single polysaccharide chains of proteoglycans was performed on columns $(1 \mathrm{~cm} \times 3 \mathrm{~cm})$ of DEAE-cellulose (Whatman DE-52), equilibrated with $50 \mathrm{~mm}$-Tris/ $\mathrm{HCl}$ buffer, $\mathrm{pH} 8$. Samples diluted to a $\mathrm{NaCl}$ concentration of less than $50 \mathrm{mM}$, mixed with internal standards of hyaluronan $(0.25 \mathrm{mg})$, chondroitin sulphate $(1.25 \mathrm{mg})$ and heparin ( $1 \mathrm{mg}$ ), were applied to the columns, which were then washed extensively first with $50 \mathrm{~mm}$-Tris/ $\mathrm{HCl}$ buffer, $\mathrm{pH} 8.0$, then with $50 \mathrm{~mm}-\mathrm{LiCl}$ in $50 \mathrm{~mm}$-sodium acetate buffer, $\mathrm{pH}$ 4.0. In this procedure an unknown labelled component, derived from UDP- $\left[{ }^{3} \mathrm{H}\right] \mathrm{GlcA}$ and recovered with the proteoglycan fraction in the initial gel-chromatography step, yet presumably unrelated to glycosaminoglycan formation (for information see Lidholt et al., 1988), was eliminated in the wash fractions (results not shown). After washing, labelled polysaccharide (proteoglycan or single glycosaminoglycan chains) was eluted at a rate of $6 \mathrm{ml} / \mathrm{h}$ with a linear salt gradient (total volume $80 \mathrm{ml}$ ) of $50 \mathrm{~mm}-1.5 \mathrm{M}-\mathrm{LiCl}$ in $50 \mathrm{~mm}$-sodium acetate buffer, pH 4.0. Fractions $(2 \mathrm{ml})$, were collected and analysed for radioactivity by liquid-scintillation counting. The glycosaminoglycan internal standards were detected by means of the carbazole reaction (Bitter \& Muir, 1962). Pooled effluent fractions were dialysed and concentrated as required before further analysis.

Free polysaccharide chains were separated with regard to molecular size by h.p.l.c. on Ultropac TSK G4000 SW columns $(0.75 \mathrm{~cm} \times 60 \mathrm{~cm}$; LKB Produkter) or Superose $6(1 \mathrm{~cm} \times 30 \mathrm{~cm}$; Pharmacia), equilibrated with $1 \mathrm{M}-\mathrm{NaCl}$ in $10 \mathrm{~mm}$-Tris/ $\mathrm{HCl}$ buffer, $\mathrm{pH}$ 7.4. Elution was performed at a flow rate of $0.5 \mathrm{ml} / \mathrm{min}$, and $1 \mathrm{ml}$ fractions were collected. The columns were calibrated with hyaluronan fractions of defined size as described previously (Lidholt et al., 1988).

Degradation of polysaccharides with $\mathrm{HNO}_{2}$ (Shively $\&$ Conrad, 1976) at pH 1.5 (deaminative cleavage at $N$ sulphated glucosamine residues), or at pH 3.9 (cleavage at $N$-unsubstituted glucosamine residues), was carried out as described previously (Thunberg et al., 1982). Deamination products were analysed on a column $(1 \mathrm{~cm} \times 190 \mathrm{~cm})$ of Sephadex G-25 (superfine grade; Pharmacia), eluted with $1 \mathrm{M}-\mathrm{NaCl}$ at a flow rate of approx. $6 \mathrm{ml} / \mathrm{h}$.

\section{RESULTS}

Previous studies on the polysaccharide chainelongation reaction in heparin biosynthesis utilized a mouse mastocytoma microsomal fraction, which was incubated with UDP- $\left[{ }^{3} \mathrm{H}\right] \mathrm{GlcA}$ and unlabelled UDPGlcNAc (Lidholt et al., 1988). The rate of chain elongation was found to vary with the concentrations of the two sugar nucleotides and was maximal when UDPGlcA and UDP-GlcNAc were added in approximately the same amounts; a molar excess of one over the other

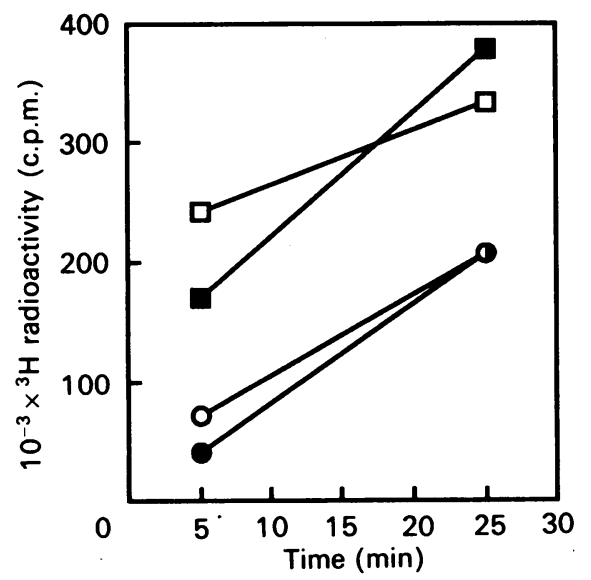

Fig. 1. Incorporation of radioactivity from UDP- $\left[^{3} \mathbf{H}\right] \mathrm{GlcA}$ into microsomal polysaccharide

Mastocytoma microsomal fraction was incubated (final volume $0.2 \mathrm{ml})$ with UDP- $\left[{ }^{3} \mathrm{H}\right] \mathrm{GlcA}$ and either $25 \mu \mathrm{M}-(\square$ and $\square$ ) or $5 \mathrm{~mm}$-UDP-GlcNAc ( $O$ and $O$ ), in the absence ( $\square$ and $O$ ) or in the presence ( $\square$ and $O$ ) of 1 mM-PAPS, as described in the Experimental section. After incubation at $37^{\circ} \mathrm{C}$ for either $5 \mathrm{~min}$ or $25 \mathrm{~min}$, labelled macromolecules were isolated by gel chromatography and quantified by scintillation counting. The values are corrected for non-specific incorporation of label (see Lidholt et al., 1988). For additional information see the text. 
precursor invariably resulted in the formation of shorter polysaccharide chains (for a discussion of possible mechanisms behind this phenomenon see Lidholt et al., 1988). During elongation the polysaccharide chains are attached to a polypeptide backbone, and the product therefore appears as a proteoglycan. No preformed glucosaminoglycan primer polysaccharide chain attached to protein could be detected in the cell-free system, and it was therefore assumed that the polysaccharide chains were formed de novo from initiation sites that contained merely the galactosyl-galactosyl-xylosyl-serine sequence that constitutes the polysaccharide-protein linkage region (Rodén, 1980). In the present investigation the effects of sulphation on chain elongation were explored by adding PAPS and the sugar nucleotides simultaneously.

\section{Effects of PAPS on chain elongation}

Simultaneous chain elongation and sulphation. Mastocytoma microcomal fraction was incubated with UDP[ $\left.{ }^{3} \mathrm{H}\right] \mathrm{GlcA}(3 \mu \mathrm{M})$ and either $25 \mu \mathrm{M}$ - or $5 \mathrm{mM}$-UDPGlcNAc, for 5 or $25 \mathrm{~min}$, as described in the Experimental section. Each incubation was performed both in the presence and in the absence of PAPS (1 mM). The ${ }^{3} \mathrm{H}$ -

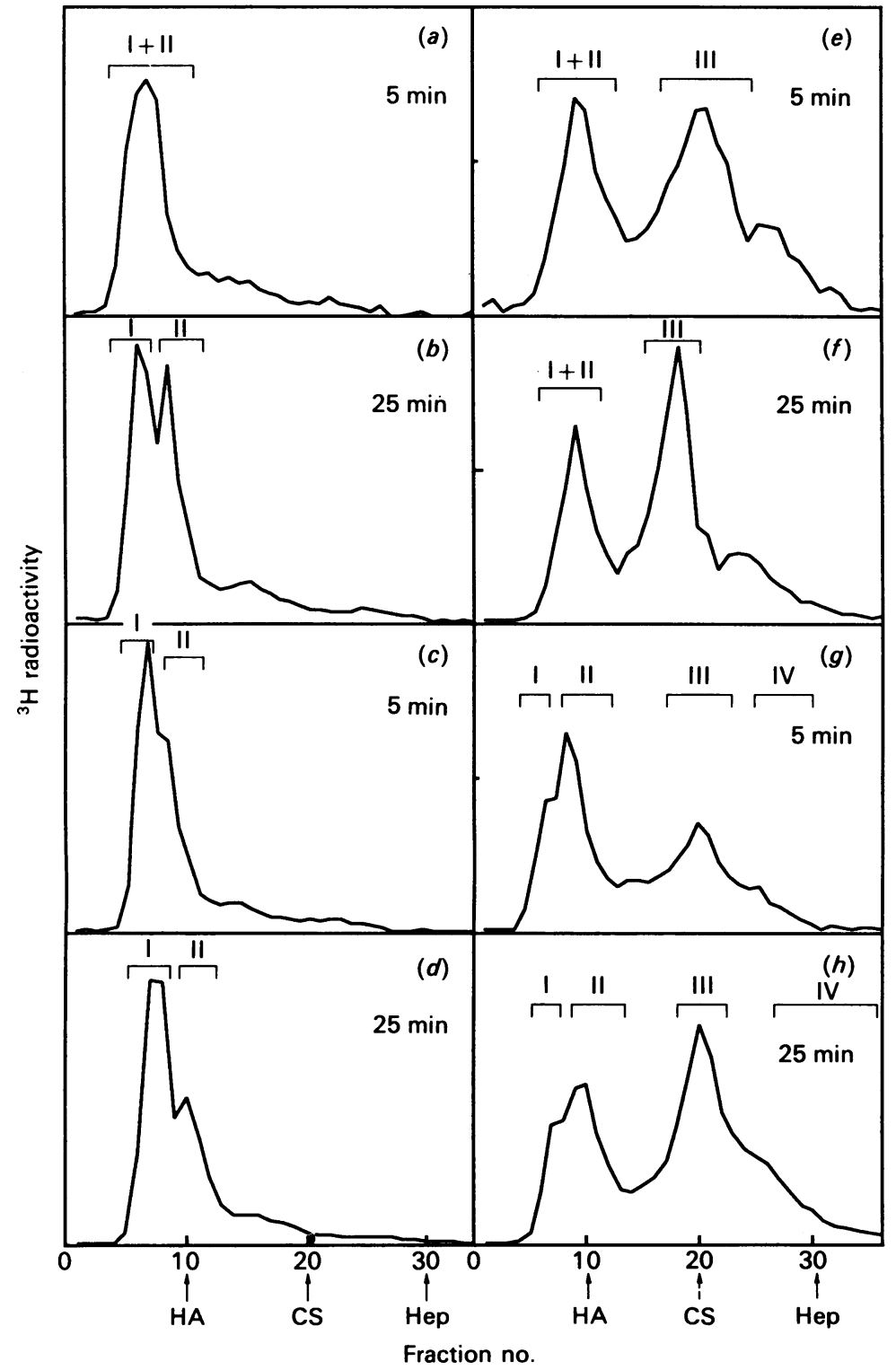

Fig. 2. Anion-exchange chromatography of non-sulphated and sulphated polysaccharide chains

Labelled proteoglycans, generated and isolated as described in Fig. 1 legend, were treated with alkali and the resulting free polysaccharide chains were separated by anion-exchange chromatography on DEAE-cellulose (see the Experimental section). Panels $(a)-(d)$, polysaccharide formed in the absence of PAPS; panels $(e)-(h)$, polysaccharide formed in the presence of PAPS; panels $(a),(b),(e)$ and $(f)$, incubations containing 5 mM-UDP-GlcNAc; panels $(c),(d),(g)$ and $(h)$, incubations containing $25 \mu \mathrm{M}$-UDP-GlcNAc; the incubation times are indicated in the panels. The arrows mark the peak elution positions of polysaccharide internal standards (HA, hyaluronan; CS, chondroitin sulphate; Hep, heparin; detected by the carbazole reaction). Fractions were pooled as indicated by the horizontal brackets, and analysed further by gel chromatography, as shown in Fig. 3. For additional information see the text. 
labelled proteoglycans formed were isolated by gel chromatography (Sephadex G-50) and quantified (Fig. 1). The incubations containing $25 \mu \mathrm{M}$-UDP-GlcNAc generated more labelled polysaccharide than did those containing $5 \mathrm{~mm}$-UDP-GlcNAc, in accord with previous findings (Lidholt et al., 1988). With more extended incubation periods at the lower UDP-GlcNAc concentration the incorporation of radioactivity into polysaccharide tended to level off, presumably owing to enzymic degradation of the labelled UDP-GlcA (see Lidholt et al., 1988). The amounts of labelled polysaccharide formed after incubation for $5 \mathrm{~min}$ in the presence of PAPS were consistently (repeat experiments; results not shown) lower than those obtained in control incubations lacking PAPS. The reason for this inhibition is unknown. No such difference was noted after longer incubation time, presumably as a result of a protective effect of PAPS on the UDP-GlcA (results not shown).

The labelled proteoglycans were treated with alkali (see the Experimental section), and the resulting free polysaccharide chains were fractionated by ion-exchange chromatography on DEAE-cellulose (Fig. 2). The nonsulphated polysaccharide formed in the absence of PAPS appeared early in the chromatograms (Figs. 2a-2d), and were generally partially separated into two fractions. Adopting a nomenclature previously defined by

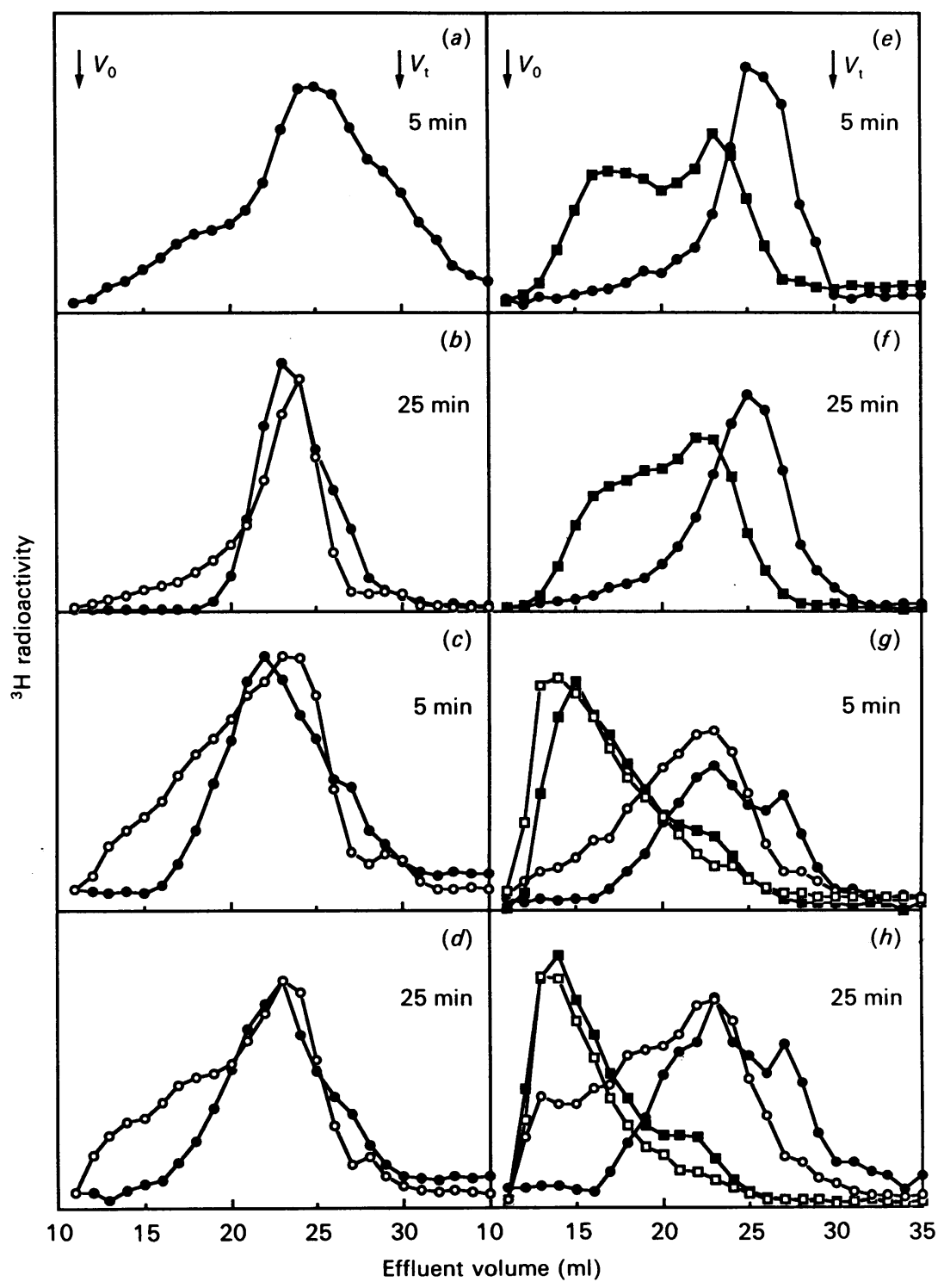

Fig. 3. Gel chromatography of non-sulphated and sulphated polysaccharide chains

Fractions of polysaccharide chains isolated by anion-exchange chromatography (see Fig. 2) were subjected to chromatography on a column of Ultropac TSK G4000 SW. Panels $(a)-(d)$, polysaccharide formed in the absence of PAPS; panels $(e)-(h)$, polysaccharide formed in the presence of PAPS; panels $(a),(b),(e)$ and $(f)$, incubations containing 5 mM-UDP-GlcNAc; panels $(c),(d),(g)$ and $(h)$, incubations containing $25 \mu \mathrm{M}$-UDP-GlcNAc; the incubation times are indicated in the panels. The samples analysed were derived from the various fractions shown in the corresponding panels of Fig. 2, such that panel $(a)$ represents the single fraction in Fig. 2(a), panel $(b)$ fractions I (O) and II $(O)$ in Fig. 2(b), panel $(c)$ fractions I (O) and II (O) in Fig. 2(c), panel $(d)$ fractions I $(O)$ and II $(O)$ in Fig. $2(d)$, panel $(e)$ fractions I + II $(O)$ and III $(\square)$ in Fig. $2(e)$, panel $(f)$ fractions I + II $(O)$ and III $(\square)$ in Fig. $2(f)$, panel $(g)$ fractions I $(O)$, II $(O)$, III $(\square)$, and IV $(\square)$ in Fig. $2(g)$, and panel $(h)$ fractions $I(O)$, II $(O)$, III ( $\square)$ and IV $(\square)$ in Fig. $2(h)$. For additional information see the text. 
Jacobsson \& Lindahl (1980) and by Riesenfeld et al. (1980), the two peaks correspond to fractions PS-NAc (peak II), i.e. the initial polymerization product composed of alternating $\mathrm{N}$-acetylglucosamine and glucuronic acid units, and PS- $\mathrm{NH}_{3}{ }^{+}$(peak I), the partially $N$-deacetylated derivative. The products generated in the presence of PAPS yielded distinct, albeit poorly resolved, peaks of sulphated material, in addition to residual non-sulphated fractions (Figs. $2 e-2 h$ ). The predominant sulphated fraction, $\mathrm{PS}^{-\mathrm{NSO}_{3}}{ }^{-}$(peak III), was previously shown to contain essentially $N$-sulphate groups only, whereas the more retarded components, PS-N/O-SO ${ }_{3}^{-}$(peak IV), would be both $\mathrm{N}$ - and $\mathrm{O}$-sulphated. Treatment of the sulphated components with $\mathrm{HNO}_{2}$ at $\mathrm{pH} 1.5$ invariably resulted in degradation of more than $80 \%$ of the labelled material to di- or oligo-saccharides (results not shown), thus demonstrating the presence of $N$-sulphate groups, as required for a heparin-related polymer. The samples isolated after $25 \mathrm{~min}$ of incubation (Figs. $2 f$ and $2 h$ ) contained relatively larger amounts of the sulphated components than did those produced after 5 min (Figs. $2 e$ and $2 g$ ).

Gel chromatography of the isolated polysaccharide chain fractions confirmed previous observations (Lidholt et al., 1988) that chains produced in the presence of $25 \mu \mathrm{M}$-UDP-GlcNAc were longer than those obtained with $5 \mathrm{~mm}$-UDP-GlcNAc (Fig. 3). This difference was particularly notable for sulphated chains, obtained after incubations performed in the presence of PAPS (compare Figs. $3 e$ and $3 f$ and Figs. $3 g$ and $3 h$ ). As shown in Fig. 2, such incubations yielded mixtures of non-sulphated and sulphated species, which were separated by ion-exchange chromatography. The gel chromatograms of the nonsulphated chains were by and large indistinguishable from those of the analogous components generated in the absence of PAPS. In contrast, the sulphated chains were much more extended, and this applied both to the $N$-sulphated fractions III and to the $N$ - and $O$-sulphated components in fractions IV. The striking differences in chain length are illustrated by the chromatograms in Fig. $3(\mathrm{~g})$, which shows the various types of polysaccharide chains isolated from a $5 \mathrm{~min}$ incubation of UDP$\left[{ }^{3} \mathrm{H}\right] \mathrm{GlcA}$ in the presence of $25 \mu \mathrm{M}$-UDP-GlcNAc and 1 mM-PAPS. Whereas the peak elution positions of the non-sulphated fractions I and II corresponded to an $M_{\mathrm{r}}$ of approx. $12 \times 10^{3}$ (as assessed by the calibration procedure described by Lidholt et al., 1988), the sulphated components appeared to have $M_{\mathrm{r}}$ in excess of $100 \times 10^{3}$, i.e. an order of magnitude difference. Similar results were obtained also when the gel chromatography was performed on agarose gel (Sepharose 2B; results not shown).

Elongation of previously sulphated chains. The marked difference in molecular size between the sulphated and non-sulphated chains noted in the above experiments raised the question as to whether further polymerization could take place on already sulphated polysaccharide. To settle this point a pulse-chase experiment was designed as outlined in the legend to Fig. 4. The mastocytoma microsomal fraction was incubated with UDP- $\left[{ }^{3} \mathrm{H}\right] \mathrm{GlcA}$

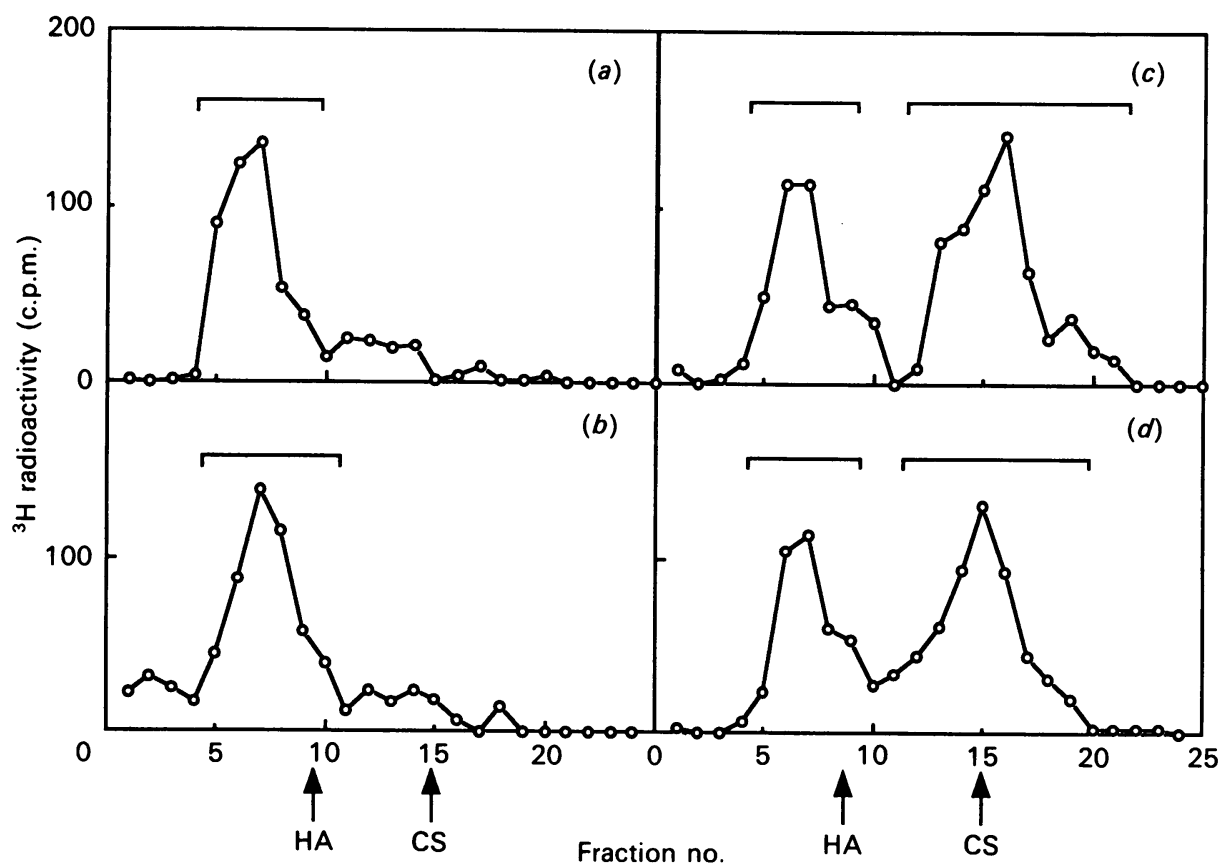

Fig. 4. Ion-exchange chromatography of pulse-chase-labelled polysaccharide chains

Mastocytoma microsomal fraction was incubated (final volume $0.5 \mathrm{ml}$ ) under standard conditions (see the Experimental section) with UDP- $\left[{ }^{3} \mathrm{H}\right] \mathrm{GlcA}$ and $5 \mathrm{~mm}$-UDP-GlcNAc, in the absence [panels $(a)$ and $\left.(b)\right]$ or in the presence [panels $(c)$ and $\left.(d)\right]$ of $1 \mathrm{~mm}$-PAPS. After $5 \mathrm{~min}, 0.25 \mathrm{ml}$ of each sample was withdrawn for analysis [panels $(a)$ and $(c)$; pulse-labelled material]. To the remaining portions was added unlabelled UDP-GlcA to a final concentration of $5 \mathrm{~mm}$, and the incubations were continued for an additional 5 min [panels $(b)$ and $(d)$ ]. Proteoglycans were isolated by gel chromatography; polysaccharide chains were released by alkali treatment and isolated by ion-exchange chromatography on DEAE-cellulose (see the Experimental section). The total amounts of ${ }^{3} \mathrm{H}$ radioactivity recovered from the samples displayed in $(a),(b),(c)$ and $(d)$ were approx. $45 \times 10^{3}$ c.p.m. Fractions were combined as indicated by the horizontal brackets and were then dialysed before further analysis by gel chromatography (Fig. 5). The less-retarded fractions in panels $(c)$ and $(d)$ are referred to in the following as non-sulphated polysaccharide and the more-retarded fractions as sulphated polysaccharide. The polysaccharide standards are as in Fig. 2. 
(approx. $3 \mu \mathrm{M}$ ) and $5 \mathrm{~mm}$-UDP-GlcNAc (yielding a low rate of polymerization; see above), in the presence or in the absence of $1 \mathrm{~mm}$-PAPS. After $5 \mathrm{~min}, 5 \mathrm{~mm}$ unlabelled UDP-GlcA was added to portions of the reaction mixtures, and incubation was continued for an additional $5 \mathrm{~min}$. The products were treated with alkali to release polysaccharide chains, which were then isolated by ionexchange chromatography (Fig. 4). The amounts of radioactivity recovered from the chase-incubated samples did not significantly differ from those of the corresponding pulse-labelled materials (see the legend to Fig. 4), indicating that the UDP-GlcA pool had been sufficiently diluted to prevent further incorporation of label during the chase. The elution positions on ion-exchange chromatography of the polysaccharide chains generated during the pulse incubations, i.e. non-sulphated chains formed in the absence of PAPS (Fig. 4a) and nonsulphated and sulphated chains formed in the presence of PAPS (Fig. 4c), remained apparently unchanged after chase (Figs. $4 b$ and $4 d$ ); moreover, the ratio of nonsulphated to sulphated components observed after pulselabelling in the presence of PAPS (Fig. 4c) was only marginally changed during the chase (Fig. 4d). Analysis of chain length by gel chromatography (Fig. 5) showed, as expected (Lidholt et al., 1988), that the non-sulphated

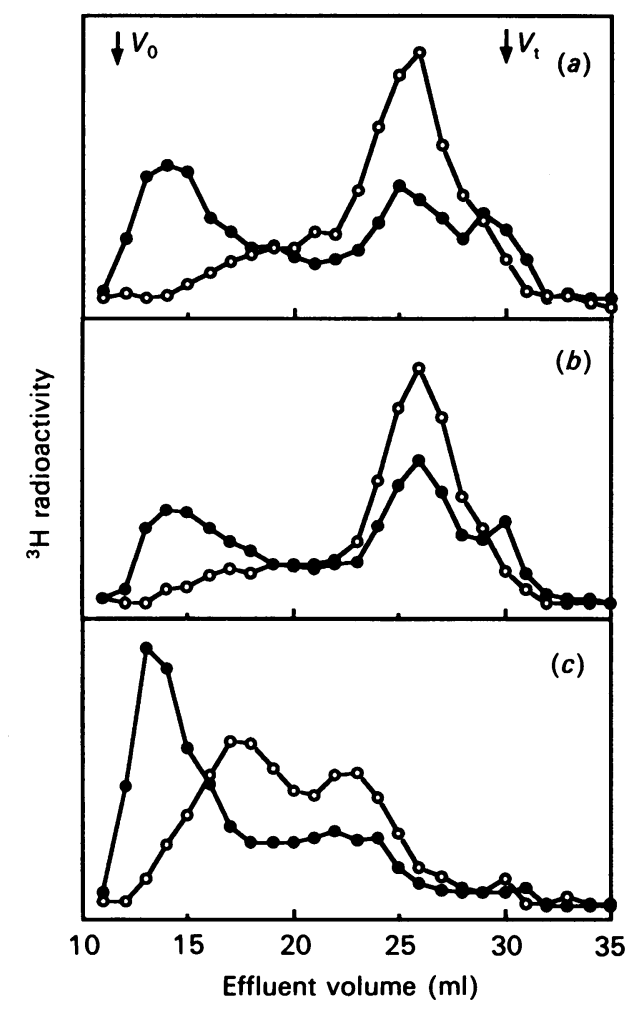

Fig. 5. Gel chromatography of polysaccharide chains isolated after pulse-chase incubation

Polysaccharide chains recovered from microsomal incubations performed in the absence [panel $(a)$ ] or in the presence [panel $(b)$, non-sulphated chains; panel $(c)$, sulphated chains] of PAPS were analysed by gel chromatography on a column of Ultropac TSK G4000 SW as described in the Experimental section. Samples isolated after pulse-labelling or after subsequent chase incubation are represented by $O$ and symbols respectively. The isolation of the various samples is described in Fig. 4. chains increased considerably in size during the chase, and this applied equally to incubations performed in the absence (Fig. $5 a$ ) or in the presence (Fig. $5 b$ ) of PAPS. In addition, however, also the sulphated pulse-labelled chains increased dramatically in size during chase incubation (Fig. 5c). These components showed a bimodal distribution after the pulse, the major peak being centred around $M_{\mathrm{r}}$ approx. $50 \times 10^{3}$, whereas the chase-incubated product had been largely shifted to an elution position corresponding to $M_{\mathrm{r}}$ approx. $200 \times 10^{3}$. The only reasonable interpretation of these findings is that, in the cell-free system under study, a sulphated polysaccharide chain remains capable of further elongation.

\section{Short-term sulphation experiments}

The experiments described above implied that polymerization is stimulated by simultaneous sulphation of the polysaccharide chain, and it was therefore of interest to define the initial sulphation events with regard to the location of target structures. The approach involved characterization of partially sulphated proteoglycans as well as polysaccharide chains, isolated after only $1 \mathrm{~min}$ of sulphation.

Proteoglycan intermediates. Mastocytoma microsomal fraction was incubated under standard conditions with UDP-[ $\left.{ }^{3} \mathrm{H}\right] \mathrm{GlcA}$, unlabelled UDP-GlcNAc $(25 \mu \mathrm{M})$ and unlabelled PAPS (0.1 mM). After 1 min labelled proteoglycan was extracted and fractionated by ion-exchange chromatography on DEAE-cellulose (Fig. 6a). Two distinct peaks were seen, one that emerged before the hyaluronan standard, at the approximate elution position of the partially $N$-deacetylated biosynthetic intermediate PS- $\mathrm{NH}_{3}^{+}$, and another, more retarded, component that appeared close to the chondroitin sulphate standard and apparently contained sulphated polysaccharide chains. Each component was recovered, treated with alkali to release free polysaccharide chains and resubmitted to ion-exchange chromatography. The non-sulphated proteoglycan fraction thus yielded virtually non-sulphated polysaccharide chains only (Fig. $6 b$ ). The more retarded proteoglycan fraction, as expected, contained mainly sulphated polysaccharide chains (Fig. 6c), which appeared at the elution position of the $N$-sulphated intermediate $\mathrm{PS}^{-\mathrm{NSO}_{3}}{ }^{-}$. However, in addition a peak of non-sulphated material, virtually indistinguishable from that derived from the non-sulphated proteoglycan, was observed. This labelling pattern, including the formation of two distinct proteoglycan species, one of which contained a mixture of sulphated and non-sulphated polysaccharide chains, was consistently noted in repeat incubations. These findings suggest that $N$-sulphation of the non-sulphated (partially $N$-deacetylated) proteoglycan precursor occurs rapidly, yet in a progressive, strictly ordered, fashion, allowing for the formation of intermediates in which a fraction of the constituent polysaccharide chains have become $N$-sulphated while others remain devoid of sulphate groups.

Polysaccharide intermediates. Since the proteoglycans isolated after $1 \mathrm{~min}$ of sulphation included species that contained a mixture of sulphated and apparently nonsulphated polysaccharide chains, it was decided to characterize those chains that served as target in the initial sulphate-transfer event. Mastocytoma microsomal fraction was incubated with UDP-[ $\left[{ }^{3} \mathrm{H}\right] \mathrm{GlcA},\left[{ }^{35} \mathrm{~S}\right] \mathrm{PAPS}$ 

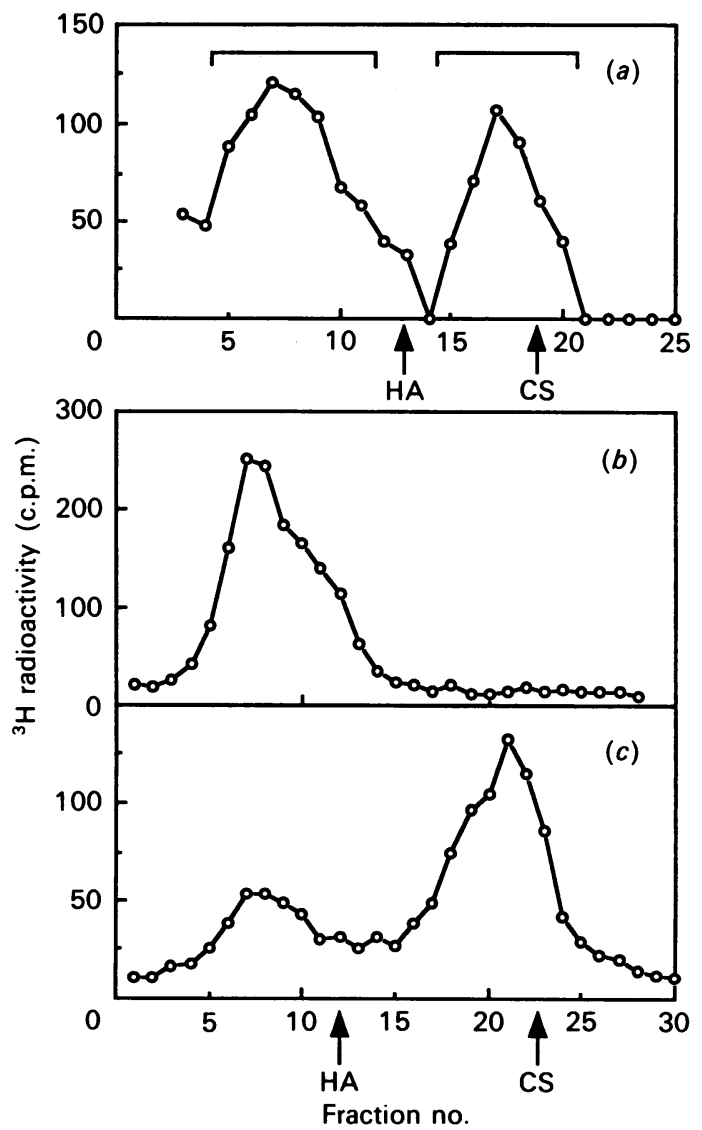

Fig. 6. Ion-exchange chromatography of proteoglycans isolated after $1 \mathrm{~min}$ of sulphation, and of their constituent polysaccharide chains

Mastocytoma microsomal fraction was incubated under standard conditions (see the Experimental section) with UDP- $\left[{ }^{3} \mathrm{H}\right]$ GlcA ( $\left.3 \mu \mathrm{M}\right)$, unlabelled UDP-GlcNAc ( $\left.25 \mu \mathrm{M}\right)$ and unlabelled PAPS (0.1 $\mathrm{mM})$ in a total volume of $2 \mathrm{ml}$ (prewarmed reagents). After $1 \mathrm{~min}$ of incubation at $37^{\circ} \mathrm{C}$ the reaction was stopped by the addition of SDS to a final concentration of $2 \%$, and the mixture was heated at $100{ }^{\circ} \mathrm{C}$ for $4 \mathrm{~min}$. Labelled proteoglycan was isolated by gel chromatography and was then subjected to ion-exchange chromatography as described in the Experimental section. The resulting fractions of less-retarded and more-retarded proteoglycan (in the following referred to as non-sulphated and sulphated proteoglycans respectively) were pooled as indicated by the horizontal brackets [panel $(a)$ ] and treated with alkali to release free polysaccharide chains. Repeated ion-exchange chromatography of the products obtained from the non-sulphated and sulphated proteoglycan fractions are shown in panels $(b)$ and $(c)$ respectively. The polysaccharide standards are as indicated in Fig. 2.

and $25 \mu \mathrm{M}$ unlabelled UDP-GlcNAc for $1 \mathrm{~min}$, and the resulting labelled polysaccharide chains were isolated as described in the legend to Fig. 7. Analysis of the products by ion-exchange chromatography showed the ${ }^{3} \mathrm{H}$ label to be distributed all over the chromatogram, but with a distinct accumulation in the region of the non-sulphated polysaccharides (before the internal hyaluronan standard in Fig. 7). This fraction also contained a minor portion of the total incorporated ${ }^{35} \mathrm{~S}$ label, most of which appeared in the more retarded elution positions of sulphated polysaccharide chains. The less retarded frac-

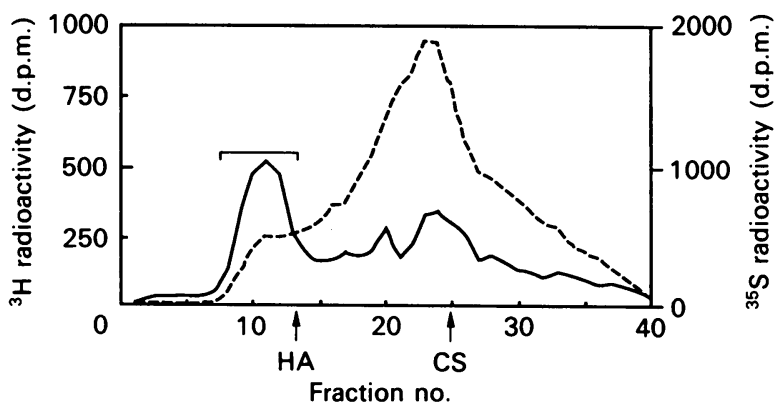

Fig. 7. Ion-exchange chromatography of polysaccharide chains isolated after $1 \mathrm{~min}$ of sulphation

Mastocytoma fraction was incubated under standard conditions (see the Experimental section) with UDP- $\left[{ }^{3} \mathrm{H}\right] \mathrm{GlcA}$ $(3 \mu \mathrm{M}),\left[{ }^{35} \mathrm{~S}\right] \mathrm{PAPS}(50 \mu \mathrm{Ci} / \mathrm{ml})$ and unlabelled UDPGlcNAc $(25 \mu \mathrm{M})$ in a total volume of $0.3 \mathrm{ml}$. After $1 \mathrm{~min}$ the reaction was interrupted, and labelled proteoglycan was isolated by gel chromatography and treated with alkali to release free polysaccharide chains. These were then fractionated by ion-exchange chromatography as described in the Experimental section. Effluent fractions were analysed for ${ }^{35} \mathrm{~S}(-. .-.-)$ and for ${ }^{3} \mathrm{H}$ ( $-(-)$ radioactivity. The fractions indicated by the horizontal brackets were combined and analysed further as shown in Figs. 8 and 9.

tion was recovered as indicated (Fig. 7) and was analysed further by gel chromatography. Both the ${ }^{3} \mathrm{H}$ and the ${ }^{35} \mathrm{~S}$ label appeared quantitatively in the excluded volume on a column of Sephadex G-25 (Fig. 8a). After treatment with $\mathrm{HNO}_{2}$ at pH 1.5 (which selectively attacks $N$ sulphated glucosamine residues; Shively \& Conrad, 1976) most of the ${ }^{35} \mathrm{~S}$ label appeared at the elution position of inorganic sulphate (Fig. $8 b$ ), indicating that the incorporated sulphate groups were essentially $N$-substituents. (The minor portion of ${ }^{35} \mathrm{~S}$ label remaining in the excluded volume after deamination was not characterized but may represent chondroitin sulphaterelated polysaccharide.) No significant amounts of ${ }^{3} \mathrm{H}$ labelled degradation products were observed (Fig. 8b), in agreement with the very low sulphate content indicated by ion-exchange chromatography (Fig. 7); the $\mathrm{N}$ sulphated glucosamine residues would presumably be too few to generate any significant amounts of small-sized oligosaccharides in the deaminative cleavage reaction. In contrast, deamination at $\mathrm{pH} 3.9$, which preferentially attacks $N$-unsubstituted glucosamine units (Shively \& Conrad, 1976), induced major degradation of the ${ }^{3} \mathrm{H}$ labelled polysaccharide backbone, indicating significant deacetylation of these units. This treatment also led to the formation of ${ }^{35} \mathrm{~S}$-labelled oligosaccharides, which were largely included in the Sephadex G-25 gel (Fig. 8c). This result demonstrated the coexistence of $N$-sulphated and $N$-unsubstituted glucosamine units in the same polysaccharide chain. Experiments in which a $1 \mathrm{~min}$ pulse-labelling with $\left[{ }^{35}\right.$ S]PAPS was followed by chase incubation with unlabelled PAPS yielded high-sulphated labelled polysaccharide that was resistant to deamination at $\mathrm{pH} 3.9$, demonstrating that the residual $N$ unsubstituted glucosamine units were accessible to $N$ sulphation (results not shown).

Analysis by gel chromatography (Superose 6) of the low-sulphated polysaccharide fraction described above 


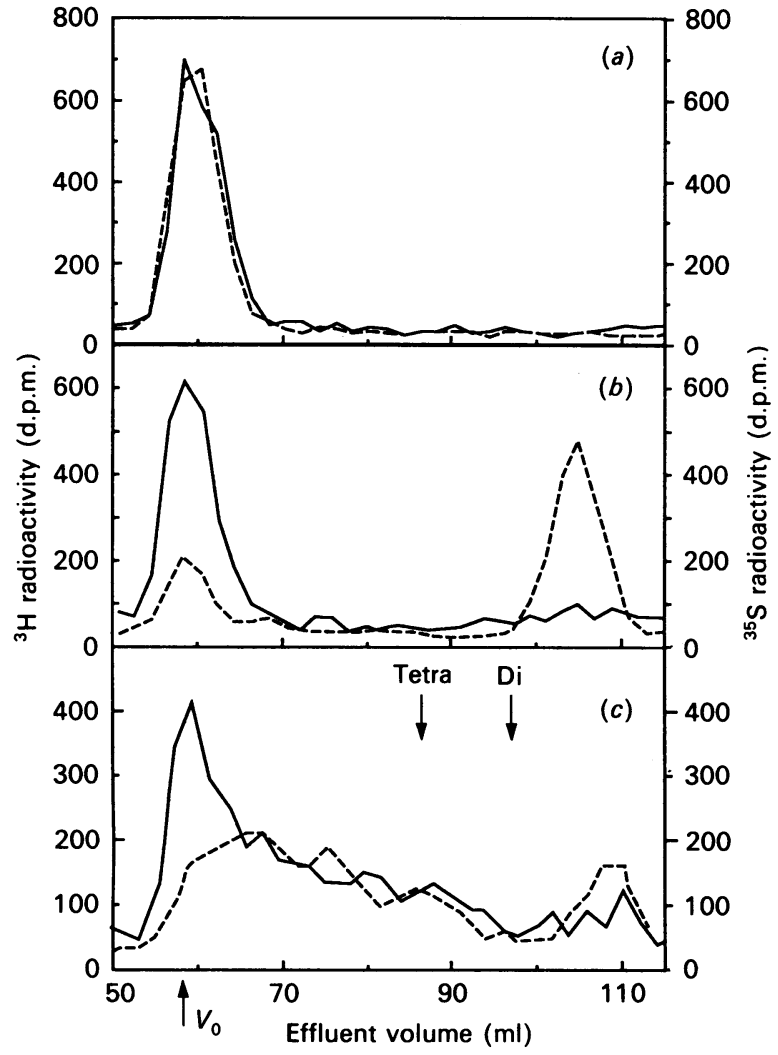

Fig. 8. Effects of $\mathrm{HNO}_{2}$ on low-sulphated chain intermediate

Low-sulphated polysaccharide chains isolated after $1 \mathrm{~min}$ of polymerization/sulphation, as illustrated in Fig. 7, were subjected to gel chromatography on a column of Sephadex G-25 without further treatment [panel $(a)$ ], after treatment with $\mathrm{HNO}_{2}$ at $\mathrm{pH} 1.5$ [panel $(b)$ ] or after treatment with $\mathrm{HNO}_{2}$ at $\mathrm{pH} 3.9$ [panel (c)]. Effluent fractions were analysed for ${ }^{35} \mathrm{~S}\left(----_{-}\right)$and for ${ }^{3} \mathrm{H}(-)$ radioactivity. The elution positions of standard di- and tetra-saccharides, isolated after deaminative cleavage of heparin, are indicated by arrows. The retarded peak of ${ }^{35} \mathrm{~S}$ radioactivity shown in panel $(c)$ probably represents inorganic sulphate, released from $N$-sulphated glucosamine units as a result of less than complete specificity of the deamination conditions. For additional information see the text.

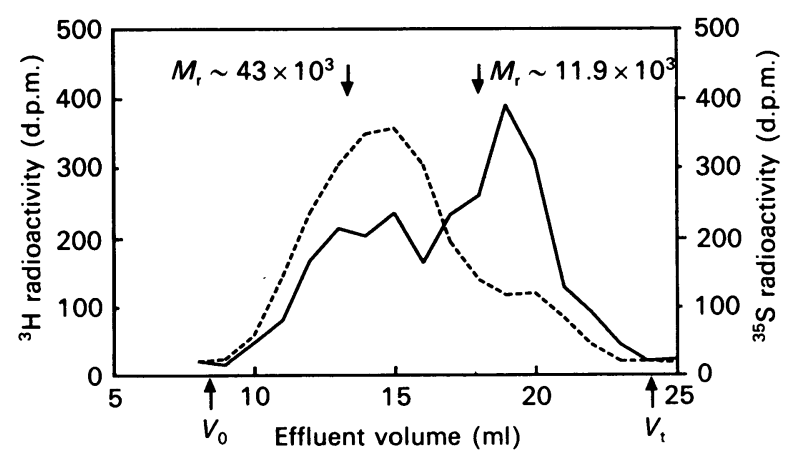

Fig. 9. Gel chromatography of low-sulphated chain intermediate

Low-sulphated polysaccharide chains isolated after $1 \mathrm{~min}$ of polymerization/sulphation, as illustrated in Fig. 7, were analysed by gel chromatography on a column of Superose 6 as described in the Experimental section. Effluent fractions were analysed for ${ }^{35} \mathrm{~S}(-. . .-)$ ) and for ${ }^{3} \mathrm{H}(-)$ radioactivity. The arrows indicate the elution positions of well-characterized hyaluronan fractions of known $M_{\mathrm{r}}$. gave the results shown in Fig. 9. The elution pattern indicated a polydisperse distribution of ${ }^{3} \mathrm{H}$-labelled polysaccharide, with two partially separated subfractions centred around $M_{\mathrm{r}} 40 \times 10^{3}$ and $9 \times 10^{3}$. Both populations of molecules also carried $\left.{ }^{35} \mathrm{~S}\right]$ sulphate groups. However, although most of the ${ }^{3} \mathrm{H}$ label occurred in the pool of smaller-sized molecules, the ${ }^{35} \mathrm{~S}$ label was predominantly accumulated in the high- $M_{\mathrm{r}}$ fraction. The initial $N$ sulphation reactions thus appear preferentially to involve the longest polysaccharide chains in the newly synthesized proteoglycan population.

\section{DISCUSSION}

Previous studies on heparin biosynthesis in cell-free systems have focused mainly on the mechanisms of polymer modification, by which non-sulphated heparinprecursor polysaccharide chains with the structure [GlcAGlcNAc $_{n}$ become converted into sulphated polymers composed of various disaccharide units arranged in complex sequences. During this process the polysaccharide chains are linked to a polypeptide backbone in a proteoglycan structure (Lidholt et al., 1988). Consideration of the kinetics and specificity of polymer modification have led to the proposal of a model of the process (Lindahl et al., 1986; Lindahl \& Kjellén, 1987), according to which proteoglycan intermediates traverse a number of membrane-bound enzyme complexes and thereby become subjected to the various modification reactions in a stepwise ordered fashion. This model accounts for the efficiency and rapidity of the overall process, completed heparin chains being detectable after less than $30 \mathrm{~s}$ of sulphation (Höök et al., 1975). However, most of the experiments behind this proposal were based on artificial segregation of the polymerization and sulphation reactions, since PAPS was added to microsomal fractions that already contained preformed labelled non-sulphated proteoglycan. Thus little information is available concerning the relationship between these two processes in the microsomal system, let alone the intact cell.

In the present study this problem was approached by using the same mastocytoma microsomal system as previously employed, except that polysaccharide chain formation and sulphation were initiated simultaneously. Again, the concerted process was remarkably effective, since proteoglycans containing sulphated polysaccharide chains could be detected after only 1 min of incubation (Fig. 6). The microsomal fraction lacked preformed polysacharide 'primers' of any appreciable size (Lidholt et al., 1988), and it is therefore assumed that the labelled chains were synthesized de novo during this incubation period. Nevertheless, polymerization and sulphation appeared to be at least partly segregated in spite of the continuous presence of PAPS, since the incubation yielded distinct fractions of sulphated and non-sulphated proteoglycan. Moreover, mixed-type proteoglycans could be demonstrated, which appeared to contain $N$ sulphated as well as non-sulphated chains bound to the same protein core, but no significant amounts of chains with intermediate sulphate contents. This macromolecule could represent an intermediate, isolated in the course of its interaction with the $N$-deacetylase- $N$-sulphotransferase enzyme complex, such that the chains substituted on the 'leading end' of the core protein had encountered both types of enzymes, whereas those on the 
'trailing end' had reacted with the $N$-deacetylase only (or had not yet reached the enzymes). These results thus are compatible with the proposed model for polymer modification.

Other results of this study, however, point to a more complex inter-relation between the polymerization and sulphation reactions. Chains synthesized in the presence of PAPS thus were considerably longer than those produced in the absence of the sulphate donor. The effect could not be simply ascribed to a stimulation of the glucuronosyltransferases and/or $\mathrm{N}$-acetylglucosaminyltransferases by PAPS, since the non-sulphated polysaccharide chains formed in the presence of PAPS were shorter than the sulphated chains and, in fact, did not differ in size from the chains produced in the absence of PAPS. Instead, the difference in chain length appears to be linked to the actual presence of sulphate groups in the target chains. Two major alternative (but not necessarily mutually exclusive) hypotheses may be advanced to explain this phenomenon. Firstly, the affinity of the polysaccharide chains for the sulphotransferase enzymes may increase with the chain length, such that significant sulphation would occur only once the chain has reached a certain critical size. Such a mechanism would result in preferential sulphation of the proteoglycan molecules that carry the longest chains; it would also be compatible with the finding that the total amounts of polysaccharide synthesized in the presence of PAPS did not differ appreciably from those obtained in the absence of PAPS (Fig. 1). Although this possibility cannot be excluded, it is noted that the chains produced in the absence of PAPS contain a rather small pool of molecules in the size range covered by the largest sulphated chains, synthesized in the presence of PAPS (Fig. 3). Alternatively, the incorporation of $N$-sulphate groups could facilitate the glycosyltransferase reactions, either by lowering the actual $K_{\mathrm{m}}$ for the enzymes or by somehow favourably influencing the physical disposition of the polysaccharide substrate. The pulse-chase experiment illustrated in Figs. 4 and 5 indicated that sulphated chains are capable of further elongation in the microsomal system [see also studies by DeLuca et al. (1973) on microsomal chondroitin sulphate synthesis], in accord with the possibility that sulphation might indeed promote subsequent polymerization.

A concept of polysaccharide chain formation that unifies the various findings of the present study features a two-stage process by which chain elongation is initiated without any significant concomitant incorporation of sulphate groups. Once above a certain (critical?) size, the chain undergoes $N$-sulphation, which then stimulates further chain elongation. It is important to note that the relationship between sulphation and polymerization studied here is restricted to the role of $N$-sulphate groups. It is well established that $N$-sulphation precedes the incorporation of $O$-sulphate groups; in accord with this notion, the sulphate groups present in the low-sulphated heparin-precursor polysaccharide isolated after $1 \mathrm{~min}$ of polymerization/sulphation appeared to be predominantly $N$-substitutents (Fig. 8). Our results do not, however, exclude a regulatory role for selected individual sulphate groups in unique positions, such as the recently discovered $O$-sulphate residue on one of the galactose moieties of the polysaccharide-protein linkage region (in chondroitin sulphate; see Sugahara et al., 1988).

This work was supported by Grants 2309 and 6525 from the Swedish Medical Research Council and Grant 600/83D98:2 from the Swedish Council for Forestry and Agricultural Research, by the National Swedish Board for Technical Development, by Kabi Vitrum A.B., Stockholm, Sweden, and by the Faculty of Veterinary Medicine, Swedish University of Agricultural Sciences. This is Paper XVII of a series in which the preceding reports are by Lidholt et al. (1988) and Kusche et al. (1988).

\section{REFERENCES}

Bitter, T. \& Muir, H. M. (1962) Anal. Biochem. 4, 330-334

DeLuca, S., Richmond, M. E. \& Silbert, J. E. (1973) Biochemistry 12, 3911-3915

Enerbäck, L., Kolset, S. O., Kusche, M., Hjerpe, A. \& Lindahl, U. (1985) Biochem. J. 227, 661-668

Furth, J., Hagen, P. \& Hirsch, E. I. (1957) Proc. Soc. Exp. Biol. Med. 95, 824-828

Höök, M., Lindahl, U., Hallén, A. \& Bäckström, G. (1975) J. Biol. Chem. 250, 6065-6071

Jacobsson, I. \& Lindahl, U. (1980) J. Biol. Chem. 255, 5094-5100

Jacobsson, I., Bäckström, G., Höök, M., Lindahl, U., Feingold, D. S., Malmström, A. \& Rodén, L. (1979) J. Biol. Chem. 254, 2975-2982

Jacobsson, I., Lindahl, U., Jensen, J. W., Rodén, L., Prihar, H. \& Feingold, D. S. (1984) J. Biol. Chem. 259, 1056-1063

Kjellén, L., Pettersson, I., Lillhager, P., Steen, M.-L., Pettersson, U., Lehtonen, P., Karlsson, T., Ruoslahti, E. \& Hellman, L. (1989) Biochem. J., in the press

Kusche, M., Bäckström, G., Riesenfeld, J., Petitou, M., Choay, J. \& Lindahl, U. (1988) J. Biol. Chem. 263, 15474-15484

Lidholt, K., Riesenfeld, J., Jacobsson, K.-G., Feingold, D. S. \& Lindahl, U. (1988) Biochem. J. 254, 571-578

Lindahl, U. (1989) in Heparin: Chemical and Biological Properties; Clinical Applications (Lane, D. A. \& Lindahl, U., eds.), pp. 159-189, Edward Arnold, London

Lindahl, U. \& Kjellén, L. (1987) in The Biology of the Extracellular Matrix: Biology of Proteoglycans (Wight, T. N. \& Mecham, R. P., eds.), pp. 59-104, Academic Press, New York

Lindahl, U., Bäckström, G., Jansson, L. \& Hallén, A. (1973) J. Biol. Chem. 248, 7234-7241

Lindahl, U., Feingold, D. S. \& Rodén, L. (1986) Trends Biochem. Sci. 11, 221-225

Riesenfeld, J., Höök, M. \& Lindahl, U. (1980) J. Biol. Chem. 255, 922-928

Riesenfeld, J., Höök, M. \& Lindahl, U. (1982) J. Biol. Chem. 257, 421-425

Robinson, H. C., Horner, A. A., Höök, M., Ögren, S. \& Lindahl, U. (1978) J. Biol. Chem. 253, 6687-6693

Rodén, L. (1980) in The Biochemistry of Glycoproteins and Proteoglycans (Lennarz, W. J., ed.), pp. 267-371, Plenum Publishing Corp., New York

Shively, J. E. \& Conrad, H. E. (1976) Biochemistry 15, 3932-3942

Silbert, J. E. (1963) J. Biol. Chem. 238, 3542-3546

Sugahara, K., Yamashina, I., De Waard, P., Van Halbeek, H. \& Vliegenthart, J. F. G. (1988) J. Biol. Chem. 263, 10168-10174

Thüberg, L., Bäckström, G., Wasteson, Å., Robinson, H. C., Ögren, S. \& Lindahl, U. (1982) J. Biol. Chem. 257, 10278-10282 